\title{
A novel gene, FAM11A, associated with the FRAXF CpG island is transcriptionally silent in FRAXF full mutation
}

\author{
Marie A Shaw ${ }^{1}$, Pietro Chiurazzi ${ }^{2}$, Dennis R Romain ${ }^{3}$, Giovanni Neri ${ }^{4}$ and Jozef Gécz ${ }^{\star 1,5}$ \\ ${ }^{1}$ Department of Cytogenetics and Molecular Genetics, Women's and Children's Hospital, Adelaide, SA, Australia; \\ ${ }^{2}$ Department of Paediatrics, University Hospital of Messina, Italy; ${ }^{3}$ Cytogenetics Laboratory, Department of \\ Laboratory Services, Wellington Hospital, Private Bag 7902, Wellington South, New Zealand; ${ }^{4}$ Istituto di Genetica \\ Medica, Universita' Cattolica, Roma, Italy; ${ }^{5}$ Department of Paediatrics, University of Adelaide, Adelaide, SA, \\ Australia
}

The cytogenetic expression of the FRAXF fragile site is due to an expanded, hypermethylated and unstable CGG repeat in Xq28. Normal individuals have 6-38 triplet repeats while individuals expressing the fragile site have expansions of greater than 300 triplets. Through analysis of the region adjacent to the fragile site, we have identified a $\sim 2.6 \mathrm{~kb}$ cDNA originating from the $F R A X F$ fragile site associated $\mathrm{CpG}$ island, and containing the unstable FRAXF CGG repeat in its $5^{\prime}$ UTR region. This gene, FAM11A, comprises at least seven exons, shows alternative splicing, and extends over $35 \mathrm{~kb}$ of genomic DNA distal to the FRAXF fragile site. Analysis of the FAM11A CDNA sequence has identified a $1050 \mathrm{bp}$ open reading frame encoding a 350 amino acid protein. We have also identified $F A M 11 B$ a highly conserved $(88 \%$ at the protein level) transcribed chromosome 2 retropseudogene. We show that the novel FRAXF fragile site associated gene $F A M 11 A$ is transcriptionally silenced in a normal individual with a cytogenetically and molecularly detectable FRAXF CGG full mutation (fragile site). Finally, we were able to reactivate FAM11A transcription by treatment of a FRAXF lymphoblastoid cell line with the demethylating agent 5azadeoxycytidine, thus demonstrating the critical role of FRAXF methylation in FAM11A silencing. European Journal of Human Genetics (2002) 10, 767 - 772. doi:10.1038/sj.ejhg.5200881

Keywords: FRAXF; FAM11A; fragile sites; transcriptional silencing; transcribed retropseudogene; FAM11B

\section{Introduction}

FRAXF is a folate sensitive fragile site in Xq28. It was originally identified in 1993 in a family with developmental delay. ${ }^{1}$ Since then, it has been seen in only a small number of additional families tested cytogenetically because of MR or developmental delay. ${ }^{1-4}$ In addition to FRAXF there are two other folate sensitive fragile sites in Xq27-28, FRAXA and FRAXE. The molecular basis of all three Xq27-28 fragile sites is an expanded, unstable, and hypermethylated CGG repeat. ${ }^{3-9}$ While FRAXA and FRAXE are associated with mental retardation, FRAXF was consid-

*Correspondence: J Gécz; Department of Cytogenetics and Molecular Genetics, Women's and Children's Hospital, 72 King William Road, North Adelaide, SA 5006, Australia. Tel: 6188161 6339; Fax: 6188161 7342; E-mail: jozef.gecz@adelaide.edu.au

Received 26 April 2002; revised 22 July 2002; accepted 24 July 2002 ered to be benign. A study by Holden et al ${ }^{10}$ suggested that the high incidence of 12 and 14 repeat alleles, with only 1/501 alleles having 13 repeats, indicated a functional significance for the FRAXF repeat region. FRAXF has been investigated as a causative factor in diseases such as mental retardation with multiple congenital anomalies, ${ }^{11}$ autism and pervasive developmental disorder, ${ }^{12}$ and PPM-X (Psychosis, Pyramidal signs, Macroordism), ${ }^{13}$ however association with any of these phenotypes is yet to be confirmed. Based on this and previous work on FRAXF ${ }^{3,4}$ it has been assumed that the FRAXF fragile site does not disturb expression of any gene, at least not at any phenotypically discernible level.

We report on the identification of a novel FRAXF fragile site associated gene FAM11A. The FAM11A gene is characterised in terms of its full length cDNA sequence, 
genomic structure, conservation, and transcriptional silencing in FRAXF full mutation.

\section{Materials and methods}

\section{Family information}

We present data from a male who expresses the FRAXF fragile site in Xq28. This individual (II:3) was initially described by Romain et $a l^{2}$ in a family without mental retardation. Parrish et $a l^{3}$ later showed that this individual (II:3, family 2) exhibited methylated FRAXF allele of $1.6 \mathrm{~kb}$. An EBV virus transformed lymphoblast cell line from this individual was established and cultured.

\section{Database searches and gene structure determination}

Genbank sequence searches were carried out with the BLAST $^{\circledR}$ search algorithm (Basic Local Alignment Search Tool) ${ }^{14}$ at the NCBI website (http://www.ncbi.nlm.nih.gov). An EST cluster, mapping to the FRAXF region, was identified from the Unigene database at NCBI. Comparison between the human Unigene cluster Hs.11522 and FRAXF genomic sequence (U71148) was used to determine the genomic structure of the FAM11A gene. DNA sequences, ESTs and Unigene clusters were downloaded and further manipulated using the Lasergene software package (DNA Star).

\section{Expression analysis of FRAXF full mutation}

Lymphoblast cells were harvested and RNA isolated from approximately $5 \times 10^{6}$ cells using TRIZOL reagent (GIBCO BRL). Approximately $1 \mu \mathrm{g}$ of purified total RNA was random primed for first-strand cDNA synthesis, using SuperScript II RNAse $\mathrm{H}^{-}$reverse transcriptase (GIBCO $\mathrm{BRL}$ ). Reverse transcription was carried out for $1 \mathrm{~h}$ at $42^{\circ} \mathrm{C}$ in a thermal cycler (Hybaid). Negative controls containing no reverse transcriptase were run at the same time. PCR was carried out using primers designed to amplify the region from exon 2 to 5 of FAM11A (E2F1: 5'-GGA TGG CAT CAT ACA GTG GAG-3', E5R1: 5'-CAA GAC GGA CCA CAC AAT GTA G-3'). EsteraseD (ESD) specific primers were used as a control for RT-PCR efficiency. ${ }^{15}$ Standard conditions for PCR were $30 \mathrm{~s}$ at $94^{\circ} \mathrm{C}, 30 \mathrm{~s}$ at $60^{\circ} \mathrm{C}$, and $30 \mathrm{~s}$ at $72^{\circ} \mathrm{C}$ for 35 cycles in a final $50 \mu$ l volume containing $100 \mathrm{mM}$ Tris- $\mathrm{HCl}, \mathrm{pH}=8.3 ; 50 \mathrm{mM} \mathrm{KCl} ; 1.5 \mathrm{~mm}$ $\mathrm{MgCl}_{2} ; 0.2 \mathrm{~mm}$ of each dNTP; 50 pmol of each primer; and $2 \mathrm{U}$ of Taq DNA polymerase (Roche). PCR products were analysed on a $1.5 \%$ agarose gel with $0.5 \mathrm{mg} \mathrm{ml}^{-1}$ ethidium bromide in $1 \times$ TBE.

\section{Reactivation of FAM11A transcriptional silencing with 5-azadeoxycytidine}

For treatment with 5-azadeoxycytidine, patient and control lymphoblast cell lines were grown in a $50 \mathrm{ml}$ flask with RPMI1640 culture medium with or without 5-azadeoxycytidine $(1 \mu \mathrm{M})$ for 7 days as previously described. ${ }^{16}$ Primers and RT-PCR conditions were as described above.

\section{Alternative splicing}

Lymphoblast cDNA was used for PCR to determine the extent of alternative splicing. PCR product was extracted from the agarose gel using the Concert Nucleic Acid Purification Kit (Invitrogen) and sequenced using BigDye Terminator ready reaction kit, according to the manufacturer's specifications (Applied Biosystems).

\section{RT - PCR and Northern blot hybridisation}

In order to confirm that the FRAXF CGG repeat was part of the FAM11A transcript, the following flanking oligonucleotide primers were designed (Figure 1), $\mathrm{F}_{\mathrm{CGG}}$ 5'-CAG GGG GCG GTG GCT CAG GTT TC-3', and R CGG $^{\prime}$-CTC AGG

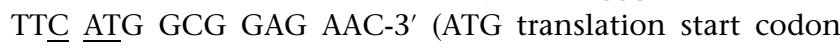
is underlined). These two primers amplify a $210 \mathrm{bp}$ product from exon 1 of FAM11A. The 3' UTR of FAM11A was amplified from lymphoblast cDNA using the primers; F: 5'-CAC CCC GCA GAC CAG AAC CAG-3', R: 5'-TCA GCA TGC CCA AAG AGA AAC TAC-3'. This 500 bp product was then used as a probe for Northern blot. A human 12 lane MultiTissue Northern blot (Clontech), containing $1 \mu \mathrm{g}$ of polyA ${ }^{+}$ RNA isolated from various human adult tissues, was hybridised with this probe using ExpressHyb solution (Clontech) as per manufacturer's instructions.

\section{Results}

Identification of a novel gene near FRAXF

Database searches using genomic sequence encompassing FRAXF fragile site (U71148) were used to identify transcribed sequences in the region around the FRAXF CGG repeat. Two Unigene clusters (Hs.352168 and Hs.11522) were identified which span the region distal to triplet. These two clusters corresponded to an as yet uncharacterised gene, which was assigned the symbol FAM11A (Family with sequence similarity $\underline{11}$, member $\underline{A}$, Genbank accession no. AF530473). Northern blot analysis indicates that FAM11A is expressed nearly ubiquitously (also supported by analysis of ESTs) with predominant expression in heart, skeletal muscle, kidney and placenta (Figure 2a). It is transcribed as a $2554 \mathrm{bp}$ message, composed of at least seven exons, encompassing about $35 \mathrm{~kb}$ of genomic sequence distal to the FRAXF CpG island. All intron/exon boundaries conform to the standard splice acceptor/splice donor consensus sequences ${ }^{17}$ (Table 1). The open reading frame (ORF) is $1050 \mathrm{bp}$ long and encodes a protein of 350 amino acids. Analysis of the cDNA sequence has identified a translation start codon within exon 1 (Figure 1). A putative transcription start site was located at position -372 with respect to the ATG start codon (Figure 1). RT-PCR analysis with primers flanking the FRAXF CGG repeat showed that the CGG repeat is transcribed as part of the FAM11A transcript (results not shown). This is also supported by the analysis of available ESTs one of which does contain the CGG repeat in its sequence (BI910833) and two others 
Table 1 Splice sites of the FAM11A gene ${ }^{a}$

\begin{tabular}{|c|c|c|c|c|}
\hline Exon & Exon size $(b p)$ & 3' splice site intron/exon & $5^{\prime}$ splice site exon/intron & Intron size $(b p)$ \\
\hline 1 & 410 & $5^{\prime}$ UTR & TCAACCCCGAG/gtgaggcggC & 20077 \\
\hline 2 & 177 & tttctcctag/TAAATТCСТC & CTCAATATCG/gtaatactgc & 2453 \\
\hline 3 & 159 & ccttttttag/AGCAGAAGGA & GTTTCCCCGGT/gtctgttgca & (4) $4587 /(4 a) 45$ \\
\hline $3 a$ & 208 & ccttttttag/AGCAGAAGGA & GTCACTAGAG/gtgagatttc & (4) $4538 /(4 a) 45$ \\
\hline 4 & 80 & ttttagttag/AAATCСТGTG & GCCCTGGCTT/gtatgtaact & 3507 \\
\hline $4 a$ & 84 & tcatttttag/TTAGAAATCC & GCCCTGGCTT/gtatgtaact & 3507 \\
\hline 5 & 176 & ccacccccag/GTTGTGTGTG & TACATTTGAG/gtaagcgttc & 625 \\
\hline 6 & 124 & ctttctttag/ATTCTGCTGG & GGAAACCACT/gtatgtact & 1298 \\
\hline 7 & 1701 & tatttttcag/GGTGGTाTGG & $3^{\prime}$ UTR & - \\
\hline
\end{tabular}

antronic sequences are shown in lower case, exonic sequences are shown in upper case.

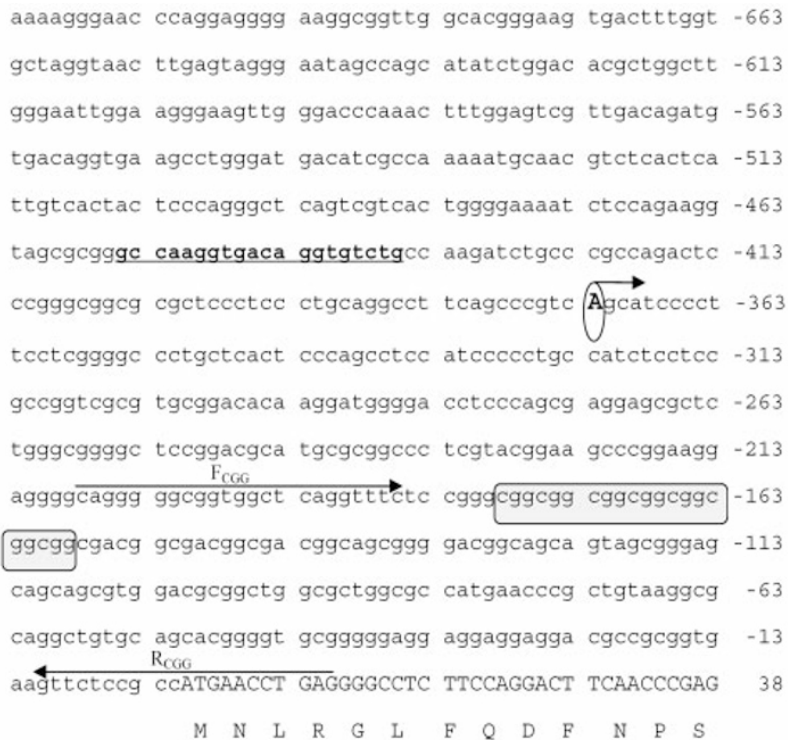

Figure 1 Genomic sequence around the putative promoter region of the FAM11A gene. The region was analysed in silico for putative promoter and transcription start. A candidate transcription start at position $-372 \mathrm{bp}$ (with respect to the translation initiation codon) has been predicted (indicated by a circle). The FRAXF (CGG) 7 repeat is boxed. There is little similarity between human (U71148) and mouse (AL672026) genomic sequence in the promoter region. The only $100 \%$ conserved stretch of sequence is that of $20 \mathrm{bp}$ highlighted (in bold and underlined). Two primers $\mathrm{F}_{\mathrm{CGG}}$ and $\mathrm{R}_{\mathrm{CGG}}$ used for PCR and RT - PCR across the FRAXF CGG repeat are shown as arrows.

(BM011526 and BM469272) which terminate just below the CGG repeat.

\section{Alternative splicing of FAM11A}

Two major FAM11A isoforms were detected on Northern blot, $\sim 2.6 \mathrm{~kb}$ and a smaller $\sim 2.1 \mathrm{~kb}$. The origin of these two isoforms is not obvious. Originally we speculated that the smaller isoform corresponds to the chromosome 2 transcribed processed retropseudogene, FAM11B transcript (see below). However, the probe as used for the Northern blot a

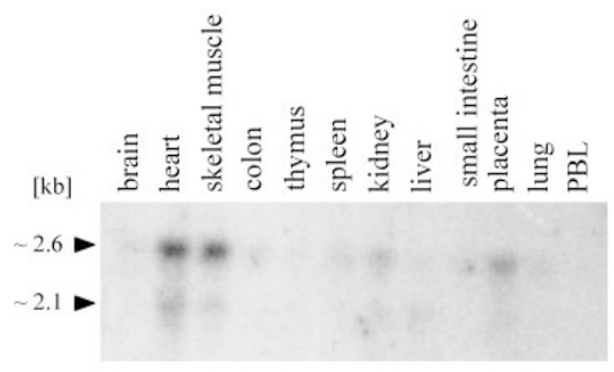

b

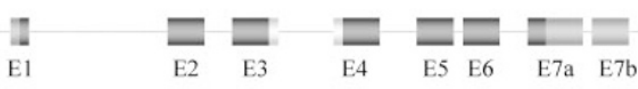

I.

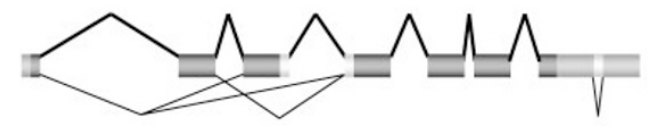

II.

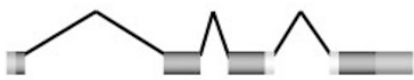

Figure 2 Analysis of the expression of the FAM11A gene. (a) Northern blot analysis of the human FAM11A gene. Human adult 12-lane multiple tissue northern blot was hybridised with a FAM11A gene $3^{\prime}$ UTR probe. Two bands at $\sim 2.6$ and $\sim 2.1 \mathrm{~kb}$ (indicated with arrowheads) of various intensity were detected across all tissues tested, however, heart, skeletal muscle, kidney and placenta were among the tissues with the highest FAM11A abundance. (b) A diagram showing alternative splicing events in FAM11A. Exons 2, 3, 4 and 7 have been found alternatively spliced. Exons ( $3 a, 4 a$ and intron 7) involved in alternative splicing are shaded light grey. The putative ORF is shaded black. I, shows the full length FAM11A transcript, while II, indicates the short FAM11A transcript truncated after exon 4.

would not cross-hybridise with the FAM $11 B$ transcript based on sequence alignment (results not shown). As a result the most likely explanation for the existence of the two major isoforms as detected by Northern blot hybridisation is alternative splicing. This was studied on human lymphoblast mRNA. Alternative splicing events were detected for exons 
2, 3, 4 and 7. An isoform missing exon 2 was detected at a low level, also exons 2 and 3 were missing in a much smaller proportion of transcripts, as determined from the RTPCR. We have observed that in a small proportion of ESTs exons 3 and 4 show alternative splicing. For exon 3 an additional $49 \mathrm{bp}$ is observed at the $3^{\prime}$ end ( $5^{\prime}$ donor splice site of intron 3), and for exon 4 an additional $4 \mathrm{bp}$ at the 5 ' end ( 3 ' acceptor splice site of intron 3 ; see Table 1 , annotated $3 \mathrm{a}$ and $4 \mathrm{a})$. An interesting alternative splicing event at the $3^{\prime}$ end of the gene occurs within exon 7 . Seventy-eight bp within exon 7 , which we call intron 7 is excluded in $\sim 50 \%$ of ESTs. RT-PCR analysis has revealed that the intron is in fact present in a higher proportion of transcripts (data not shown) and is the most predominant form. This results in a splitting of exon 7 so we have annotated this as exons $7 \mathrm{a}$ and $7 \mathrm{~b}$ (see Figure $2 \mathrm{~b}$ ).

Additionally, comparison of the two FAM11A associated Unigene clusters Hs.352168 and Hs.11522 revealed that ESTs from cluster Hs.352168 have different 3' end starting from the end of exon 4. In five of these ESTs (BM458996, BG721461, BF734532, AV720618 and BG565086) the donor splice site of exon 4 is ignored. This results in the inclusion of an extra $\sim 600 \mathrm{bp}$ of the FAM11A intron 3 sequence in the FAM11A transcript and as a consequence premature termination of transcription (see Figure 2).

\section{FAM11A is transcriptionally silenced by FRAXF full mutation}

To investigate whether FAM11A transcription is silenced by the FRAXF full mutation, lymphoblast mRNA from an individual expressing the fragile site was analysed by RT-PCR (Figure 3a). The result of this experiment shows that expansion of the FRAXF CGG repeat and consequent methylation of the CpG island results in the transcriptional silencing of the FAM11A gene.

To confirm that methylation of the FRAXF CpG island was directly involved in the transcriptional silencing of the FAM11A gene we treated the patient lymphoblastoid cell line with the demethylation agent 5-azadeoxycytidine. Reactivation of the FAM11A gene transcription as detected by RT-PCR occurred upon treatment with 5-azadeoxycytidine for 7 days (Figure 3b). This demonstrated that methylation of the FRAXF CpG island as a consequence of the CGG repeat expansion has a crucial role in the FAM11A gene silencing.

Identification of a chromosome 2 transcribed, processed retropseudogene

dbEST database searches using FRAXF genomic sequence revealed additional ESTs (Unigene cluster Hs.44680), which correspond to a highly similar gene to FAM11A on chromosome 2q21.2. Comparison of FAM11A and the chromosome 2 gene, FAM11B (Family with sequence similarity 11, member $\underline{B}$, Genbank accession no. AF530474) revealed that the chromosome 2 gene is a transcribed retropseudogene. Genes a
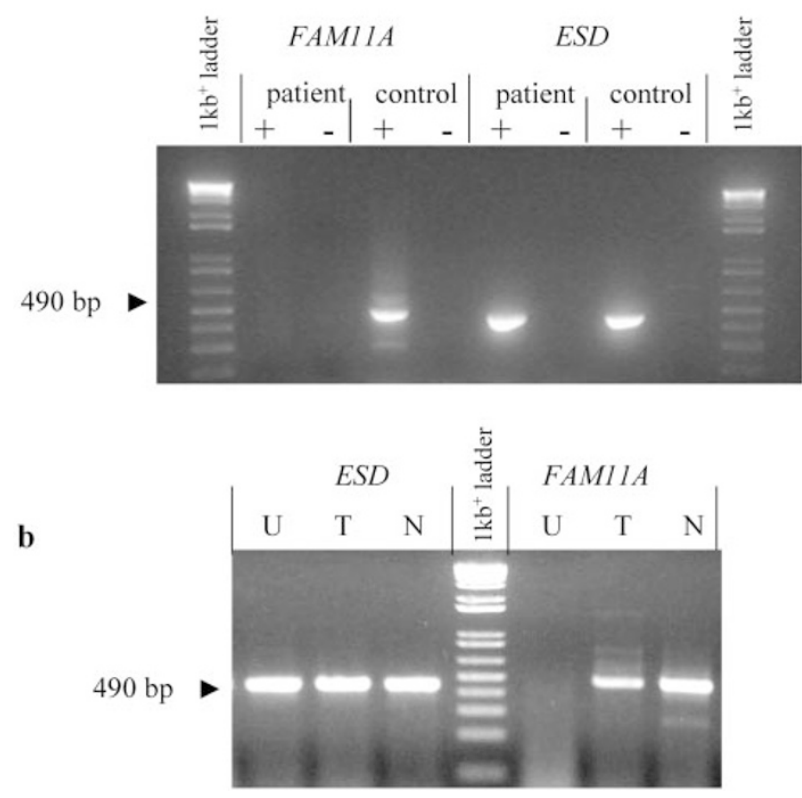

Figure 3 (a) RT-PCR analysis of the FAM11A gene in lymphoblast cells of a FRAXF normal and FRAXF full mutation individual. FAM1 $1 A$ expression was not detected in the individual with FRAXF full mutation. (b) Reactivation of FAM11A expression using 5-azadeoxycytidine in a FRAXF normal and full mutation individual. Full mutation cell lines were either treated $(T)$ with 5azadeoxycytidine or not treated $(U)$ before RT - PCR, normal cell line $(\mathrm{N})$ was not treated. FAM11A transcription is reactivated in the treated cell line. $1 \mathrm{~kb}^{+}$molecular marker (GIBCO BRL) is indicated. As control ESD primer set was used for both experiments.

duplicated by retroposition (whereby RNA is reverse transcribed into a DNA copy and inserted into the genome) which remain active, often with amino acid changes or altered tissue expression patterns, are termed 'retropseudogenes' or 'retroxaptonuons'. ${ }^{18}$ FAM11B does not contain any of the FAM11A introns. The open reading frame (ORF) of the FAM11B is also $1050 \mathrm{bp}$ long and encodes a protein of 350 amino acids, $88 \%$ identical to the FAM11A (Figure 4). The two genes, FAM11A and FAM11B, otherwise differ markedly in their $5^{\prime}$ and $3^{\prime}$ untranslated regions (results not shown).

\section{Conservation of FAM11A in M. musculus, D. melanogaster} and $C$. elegans

Database searches of mouse ESTs revealed presence of two clusters, Mm.56701 (87 ESTs) and Mm.24569 (55 ESTs). Comparison with the FAM11A gene and FAM11B retropseudogene sequences showed that the mouse Unigene cluster Mm.24569 corresponds to the mouse orthologue of FAM11A and Mm.56701 to the mouse retropseudogene. This is further supported by matches against the mouse genomic sequences, AL672026 and AC100135 (X-chromosome genomic sequence) and AC100024 (mouse 


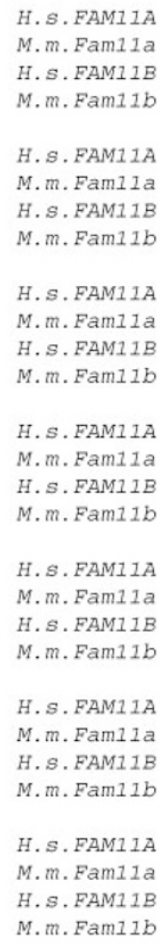

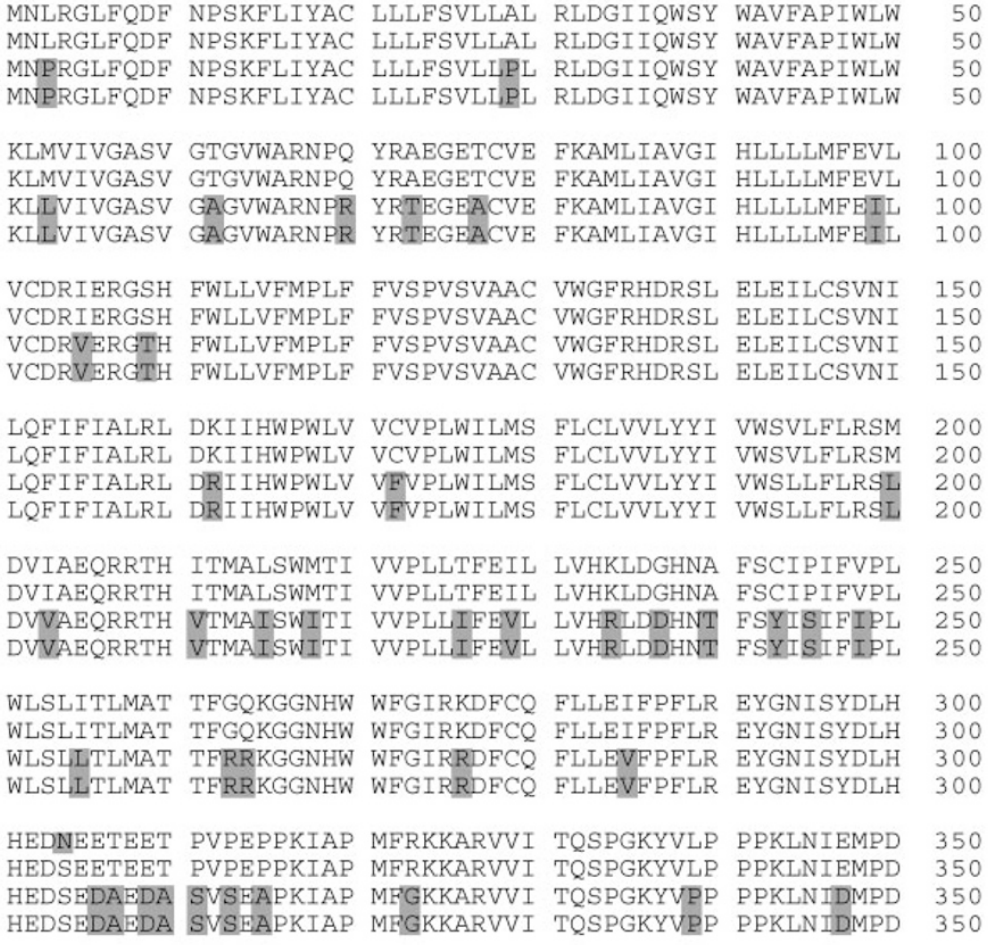

Figure 4 ClustalW multiple protein alignment of the human and mouse FAM11A and FAM11B proteins. Amino acids shaded in black are those that differ from the consensus. Exon/intron boundaries are indicated by arrowheads. All exon/exon boundaries were $100 \%$ conserved between the human and mouse genes.

chromosome 1, unplaced), respectively. Interestingly, in mouse (likewise in man), both gene Fam11a and retropseudogene Fam 11 b are transcribed. ClustalW alignment of the putative human and mouse gene and retropseudogene proteins is shown in Figure 4 . While the human and mouse proteins differ in only one amino acid, the retropseudogene-encoded proteins are 100\% identical. This high conservation of both gene and retropseudogene encoded proteins between man and mouse provides an indication of an important, conserved function. FAM11A appears to be highly conserved, with orthologues in other species including D. melanogaster (AAF48931, 36\% similarity) and C. elegans (CAB55145, 26\% similarity).

\section{Discussion}

Expansion of $F R A X F$ was initially described in a family with developmental delay. ${ }^{1}$ Since then it was reported only in a small number of additional cases. ${ }^{3}$ In contrary to the other two more proximal Xq27.3-q28 folate sensitive fragile sites, FRAXA and FRAXE, FRAXF was considered benign. This was primarily because of the absence of any FRAXF associated phenotype, but also because of a failure to identify any FRAXF associated gene(s). ${ }^{3,4}$

We report here on the identification of a novel gene, FAM11A, originating from the FRAXF CpG island and containing the FRAXF CGG repeat in its $5^{\prime}$ untranslated region. Similar to the other folate sensitive fragile site associated genes, ie FMR1/FRAXA, ${ }^{19}$ FMR2/FRAXE ${ }^{8,9}$ and FMR3/ $F R A X E{ }^{20}$ the FRAXF CGG repeat is transcribed (but not translated). We have also demonstrated that the paradigm of expansion-methylation-transcription silencing seen for the other folate sensitive fragile site associated genes is seen also for the FAM11A gene at FRAXF. However, as only one $F R A X F$ full mutation was available for testing this paradigm needs to be further confirmed on additional cases. It also remains to be tested whether FRAXF CGG expansion and transcriptional silencing of FAM11A can be associated with developmental delay as seen in some FRAXF families studied.

At least for FRAXE and FMR2, there are now several families known where full mutation does not cause developmental delay $\left(\mathrm{IQ}<70\right.$ ) (for review see $\mathrm{Gécz}^{21}$ ). When this model is applied for FRAXF, we may speculate that absence of FAM11A does not necessarily lead to developmental delay, but might in combination with other, yet unknown factors. Otherwise, the lack of the FAM11A protein is either disposable (redundant) or its function can be complemented by the highly similar chromosome 2 retropseudogene product, FAM11B. Comparison of FAM11A and FAM11B proteins shows 88\% identity. More 
interestingly, when respective gene and retropseudogene proteins are compared between man and mouse, 99.7\% (one amino acid difference) and $100 \%$ conservation is detected. There are numerous retropseudogenes in the human genome, some of which are transcribed. ${ }^{22,23}$ Their function is not known, but it has been suggested that they may be acquired during evolution as novel genes. ${ }^{18,23,24}$ Their protein products may even be functionally indistinguishable from the original gene.

In summary we have identified the gene FAM11A associated with the $F R A X F$ fragile site. Its transcription is extinguished by the FRAXF full mutation. In the absence of any $F R A X F$ associated phenotype we can only speculate about the normal role of this gene.

\section{Acknowledgements}

We thank the members of the family studied for participation, and $C$ Derwas and S MacDonald for help with cell culture. This work was supported by the National Health and Medical Research Council of Australia.

\section{References}

1 Hirst MC, Barnicoat A, Flynn G et al: The identification of a third fragile site, FRAXF in Xq27-q28 distal to both FRAXA and FRAXE. Hum Mol Genet 1993; 2: 197-200.

2 Romain DR, Chapman CJ: Fragile site Xq27.3 in a family without mental retardation. Clin Genet 1992; 41: 33-35.

3 Parrish JE, Oostra BA, Verkerk AJ et al: Isolation of a GCC repeat showing expansion in FRAXF, a fragile site distal to FRAXA and FRAXE. Nature Genet 1994; 8: 229-235.

4 Ritchie RJ, Knight SJ, Hirst MC et al: The cloning of FRAXF: trinucleotide repeat expansion and methylation at a third fragile site in distal Xqter. Hum Mol Genet 1994; 3: 2115 -2121.

5 Kremer EJ, Pritchard M, Lynch M et al: Mapping of DNA instability at the fragile $X$ to a trinucleotide repeat sequence $p(C C G) n$. Science 1991; 252: 1711-1714.

6 Oberle I, Rousseau F, Heitz D et al: Instability of a 550-base pair DNA segment and abnormal methylation in fragile $\mathrm{X}$ syndrome. Science 1991; 252: 1097-1102.

7 Yu S, Pritchard M, Kremer E et al: Fragile X genotype characterized by an unstable region of DNA. Science 1991; 252: 1179-1181.

8 Gécz J, Gedeon AK, Sutherland GR, Mulley JC: Identification of the gene FMR2, associated with FRAXE mental retardation. Nat Genet 1996; 13: $105-108$.
9 Gu Y, Shen Y, Gibbs RA, Nelson DL: Identification of FMR2, a novel gene associated with the FRAXE CCG repeat and CpG island. Nat Genet 1996; 13: 109-113.

10 Holden JJ, Walker M, Chalifoux M, White BN: Trinucleotide repeats at the FRAXF locus: frequency and distribution in the general population. Am J Med Genet 1996; 64: 424-427.

11 Vianna-Morgante AM, Mingroni-Netto RC, Barbosa AC, Otto PA, Rosenberg C: FRAXF in a patient with chromosome 8 duplication. J Med Genet 1996; 33: 611-614.

12 Holden JJ, Wing M, Chalifoux $\mathrm{M}$ et al: Lack of expansion of triplet repeats in the FMR1, FRAXE, and FRAXF loci in male multiplex families with autism and pervasive developmental disorders. Am J Med Genet 1996; 64: 399-403.

13 Lindsay S, Splitt M, Edney S et al: PPM-X: a new X-linked mental retardation syndrome with psychosis, pyramidal signs, and macroorchidism maps to Xq28. Am J Hum Genet 1996; 58: $1120-1126$.

14 Altschul SF, Gish W, Miller W, Myers EW, Lipman DJ: Basic local alignment search tool. J Mol Biol 1990; 215: 403-410.

15 Gécz J, Bielby S, Sutherland GR, Mulley JC: Gene structure and subcellular localization of FMR2, a member of a new family of putative transcription activators. Genomics 1997; 44: 201-213.

16 Chiurazzi P, Pomponi MG, Pietrobono R et al: Synergistic effect of histone hyperacetylation and DNA demethylation in the reactivation of the FMR1 gene. Hum Mol Genet 1999; 8: 2317-2323.

17 Shapiro MB, Senapathy P: RNA splice junctions of different classes of eukaryotes: sequence statistics and functional implications in gene expression. Nucleic Acids Res 1987; 15: 7155-7174

18 Brosius J, Gould SJ: On 'genomenclature': a comprehensive (and respectful) taxonomy for pseudogenes and other 'junk DNA'. Proc Natl Acad Sci USA 1992; 89: 10706-10710.

19 Verkerk AJ, Pieretti M, Sutcliffe JS et al: Identification of a gene (FMR-1) containing a CGG repeat coincident with a breakpoint cluster region exhibiting length variation in fragile $\mathrm{X}$ syndrome. Cell 1991; 65: $905-914$.

20 Gécz J: FMR3 is a novel gene associated with FRAXE CpG island and transcriptionally silent in FRAXE full mutations. J Med Genet 2000; 37: 782-784.

21 Gécz J: The FMR2 gene, FRAXE and non-specific X-linked mental retardation: clinical and molecular aspects. Ann Hum Genet 2000a; 64: 95-106.

22 Venter JC, Adams MD, Myers EW et al: The sequence of the human genome. Science 2001; 291: 1304-1351.

23 Brosius J, Venter JC, Adams MD et al: Many G-protein-coupled receptors are encoded by retrogenes. Trends Genet 1999; 15: 304-305.

24 Kuryshev VY, Skryabin BV, Kremerskothen J et al: Birth of a gene: locus of neuronal BC200 snmRNA in three prosimians and human BC200 pseudogenes as archives of change in the Anthropoidea lineage. J Mol Biol 2001; 309: 1049-1066. 